\title{
Étude expérimentale des écoulements diphasiques pétroliers en terrain accidenté
}

\author{
Experimental study of two-phase petroleum flow \\ over rough ground
}

\author{
Jacques Corteville \\ Institut Français du Pétrole \\ Département «Techniques \\ de Production en Mer » \\ Rueil-Malmaison
}

\author{
Menno Grouvel \\ Société Nationale \\ Elf-Aquitaine (Production) \\ Département « Exploitation » \\ Paris
}

\author{
Michelle Grossin \\ Compagnie Française \\ des Pétroles \\ Département « Exploitation » \\ Paris
}

\section{Objectifs}

L'êvacuation par pipeline en régime d'écoulement diphasique des bruts et gaz à condensats comporte de nombreux avantages en mer profonde, en comparaison du procédé classique de séparation en mer qui exige la recompression et l'évacuation du gaz par pipeline et celle de l'huile par un deuxième pipeline ou par tankers. Le transport diphasique économise donc beaucoup d'énergie en utilisant la pression motrice des gisements pour l'évacuation de ces effluents, et réduit notablement les coûts d'investissement et de fonctionnement en mer.

Malheureusement, les méthodes actuelles de détermination des régimes et pertes de charge en écoulement diphasique restent très imprécises, notamment pour les trajets comportant des pentes, même très faibles ; de plus, par manque d'expérience pratique, les systèmes de contrôle et les procédures d'exploitation en diphasique sont inadaptés. Pour ces raisons, le transport diphasique sur grande distance n'est qu'exceptionnellement utilisé.

Dans le cadre du Groupement Européen des Recherches Technologiques sur les Hydrocarbures, la Compagnie Française des Pétroles, 1'Institut Français du Pétrole et la Société Nationale Elf-Aquitaine (Production) ont donc constitué un groupe de recherche sur les écoulements diphasiques, qui s'est fixé pour objectifs :

- d'améliorer la connaissance pratique des écoulements diphasiques par l'observation des phénomènes et la mise au point d'appareillages de mesures adaptés; - de développer de nouvelles méthodes de calcul plus précises, basées sur une analyse théorique approfondie et une expérimentation en conditions de similitude avec les installations pétrolières.

Ces recherches, commencées en 1974, s'effectuent en collaboration avec le Laboratoire de Mécanique Expérimentale des Fluides de Paris-Orsay.

\section{Installation expérimentale}

L'analyse dimensionnelle a défini les caractéristiques principales de l'installation expérimentale. La nature des fluides, leurs vitesses, les pressions et températures ainsi que les pentes sont similaires aux conditions d'exploitation pétrolière. Le diamètre de la conduite d'essai est de $6^{\prime \prime}:$ il réduit suffisamment l'influence de la tension interfaciale, prépondérante en petits diamètres, et n'est pas très éloigné des conditions d'exploitation envisagées (6" à $18^{\prime \prime}$ environ).

La boucle diphasique a été réalisée en 1976 à Boussens dans le périmètre de l'usine de dégazolinage de S.N.E.A. (P) qui traite la production des gisements de gaz locaux. Elle permet la circulation en boucle fermée de gaz naturel et d'huile suivant des débits prédéterminés. Pour éviter les couplages entrée-sortie et stabiliser les conditions de mesure, elle comporte plusieurs séparateurs de grand volume et grande inertie thermique, des systèmes de régulation de pression, débits et températures, ainsi que des tuyères fonctionnant en régime sonique à l'admission du gaz. L'installation est conçue pour une pression de service de 65 bars et est conforme aux normes de sécurité pétrolière. Les caractéristiques principales sont résumées tableau I et figure 1.

\begin{tabular}{|c|c|}
\hline \multicolumn{2}{|c|}{ Diamètre intérieur de la conduite : $6^{\prime \prime}(146,3 \mathrm{~mm})$} \\
\hline Longueur inclinable & $: 85 \mathrm{~m}$ \\
\hline Gamme des pentes & $:$ de $-10 \%$ à $+10 \%$ \\
\hline Gamme des pressions & : de 10 à 50 bars \\
\hline Gamme des vitesses du gaz & $: 0$ à $10 \mathrm{~m} / \mathrm{s}$ \\
\hline Gamme des vitesses de I'huile & $: 0$ à $3 \mathrm{~m} / \mathrm{s}$ \\
\hline Gamme des températures & $: 10^{\circ} \mathrm{C}$ à $30^{\circ} \mathrm{C}$ \\
\hline
\end{tabular}




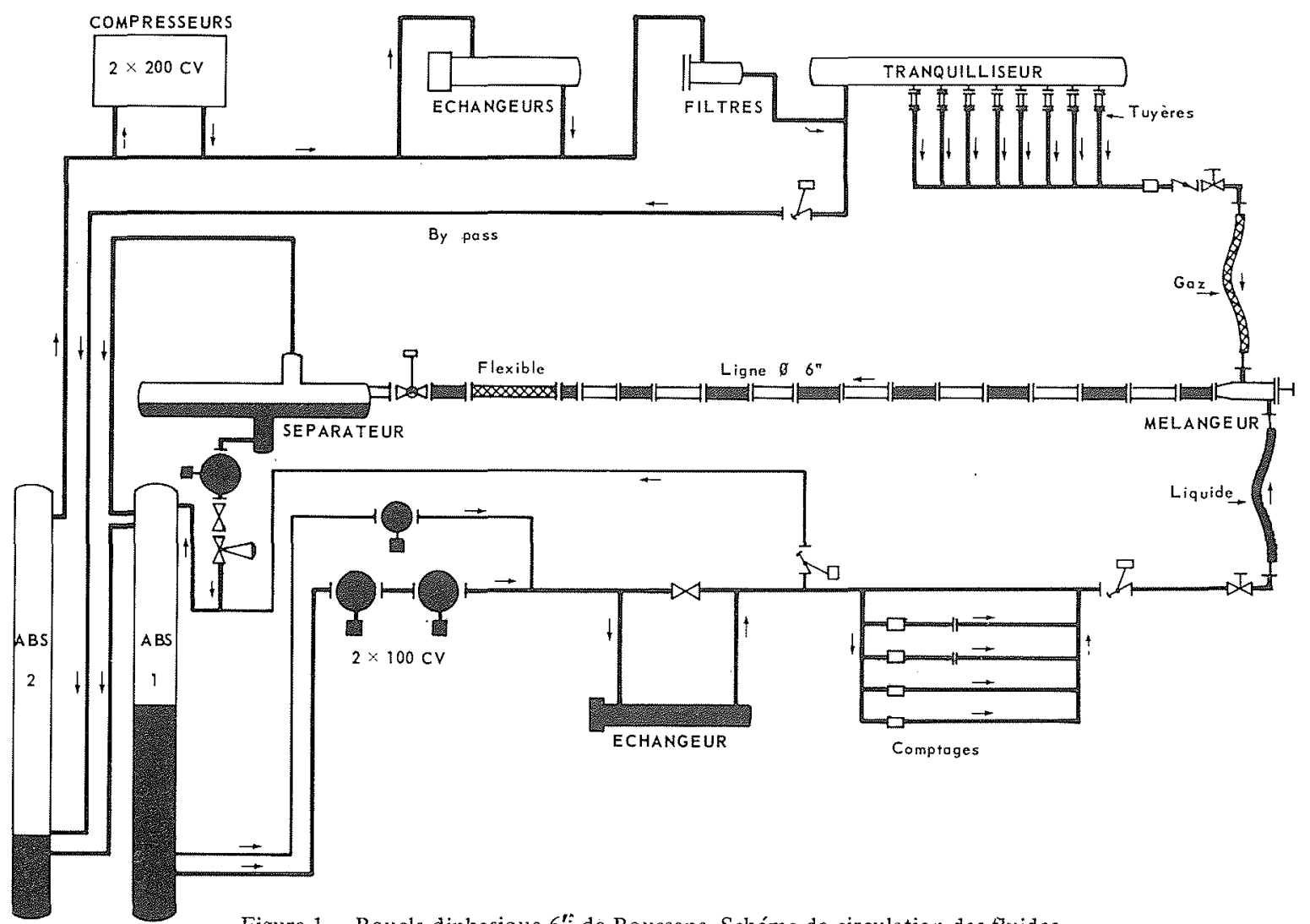

Figure 1 - Boucle diphasique $6^{\prime *}$ de Boussens. Schéma de circulation des fluides.

\section{Mode de fonctionnement de la boucle}

La boucle comporte essentiellement:

\section{a) La conduite d'essai (Fig. 2)}

Celle-ci est constituée de 13 tronçons de $9 \mathrm{~m}$ de tubes d'acier, schédule 80 , à tolérance de $\pm 1 \mathrm{~mm}$ sur le diamètre intérieur et de $0,5 \%$ sur l'ovalisation. Des brides d'assemblage particulières assurent le centrage et la continuité de ces tronçons. Deux tronçons courts $(1,3 \mathrm{~m})$ sont intercalés et contiennent les principales prises de mesure.

La partie inclinable de la conduite est fixée par des étriers et sabots de réglage d'alignement sur une rampe

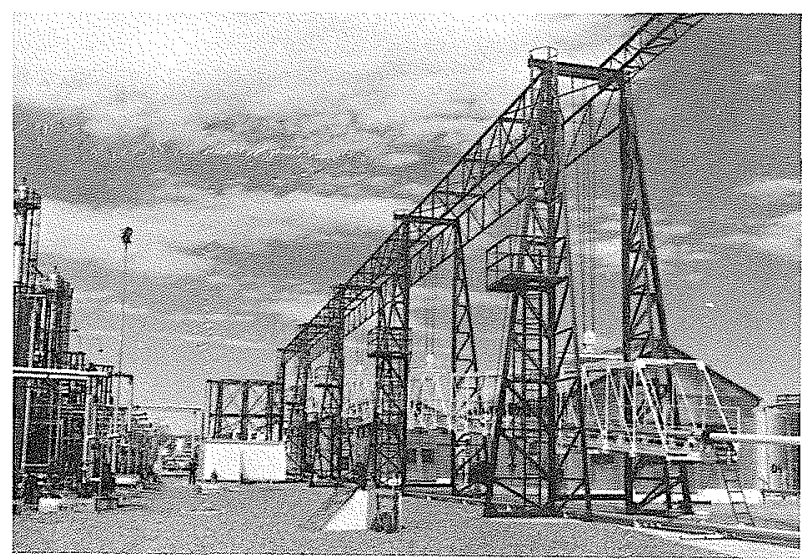

Figure 2 - Vue générale de la conduite d'essai et de sa charpente de supportage. articulée qui supporte également les chemins de câblage, les capteurs, les conduites de prises de pression. Un ensemble de portiques en treillis métallique reliés au sommet par une poutre rigide supporte la rampe inclinable et permet, au moyen de treuils, de régler la pente de la conduite sans effectuer son démontage.

Un flexible COFLEXIP de $30 \mathrm{~m}$, enroulé suivant une demie spirale le long d'un bâti métallique octogonal, assure enfin la liaison de la conduite d'essai au séparateur d'arrivée quelles que soient les pentes imposées.

\section{b) Le système d'alimentation et comptage des fluides (Fig. 3)}

Deux pompes centrifuges de $200 \mathrm{~m}^{3} / \mathrm{h}$ assurent la circulation de l'huile. Trois vannes de contrôle automa-

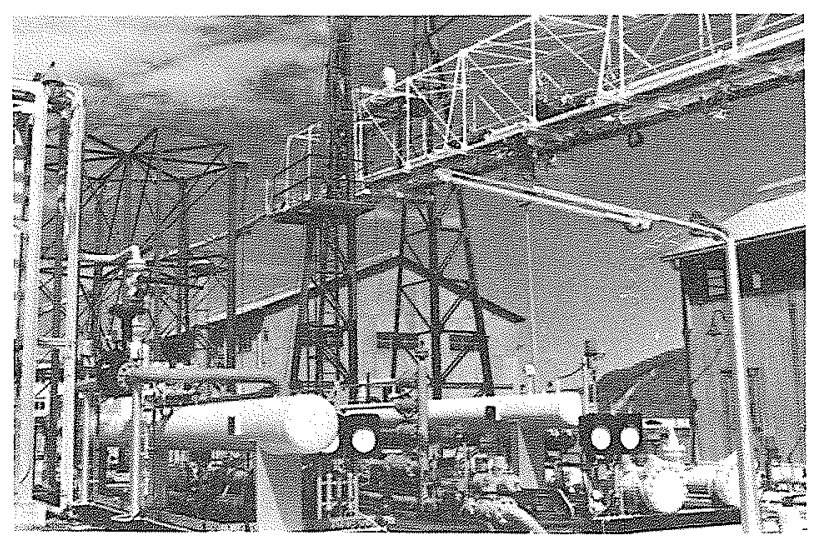

Figure 3 - Unité de conditionnement et comptage des fluides. 
tisées, agissant sur la pression de refoulement et le recyclage, permettent d'ajuster le débit dans la conduite de mesure. Les débits sont mesurés simultanément par des jeux de turbines et d'orifices calibrés. Une pompe doseuse prend le relai des pompes centrifuges dans le domaine des petits débits.

Le gaz, recomprimé par 2 compresseurs à pistons de $200 \mathrm{ch}$, est admis dans une chambre de tranquillisation où sa pression est régulée. Un jeu de 8 tuyères à cols soniques permet d'ajuster ensuite le débit qui est mesuré par un débitmètre à vortex et un système à orifices interchangeables.

L'huile et le gaz sont injectés dans la conduite d'essai par un mélangeur muni de perforations obturables de manière à créer un mélange homogène quel que soit le débit de chacune des phases.

\section{c) Le système de séparation}

L'effluent diphasique subit une séparation primaire dans un séparateur "pomme d'arrosoir" qui amortit les fluctuations de pression. Une pompe centrifuge renvoie ensuite l'huile vers une tour de $21 \mathrm{~m}^{3}$ où s'effectue la séparation fine.

Le gaz transite dans la partie supérieure de cette tour, puis est asséché mécaniquement dans une autre tour de $21 \mathrm{~m}^{3}$ avant recompression.

\section{Instrumentation}

\section{a) Instrumentation "process" (Fig. 4)}

Elle comporte environ 40 capteurs industriels classiques destinés aux mesures et régulations des débits, pressions, températures et niveaux des séparateurs. Les informations, centralisées dans la salle de contrôle, sont visualisées sur un tableau synoptique qui comporte les indicateurs et commandes permettant d'ajuster les paramètres de fonctionnement. Des enregistreurs graphiques permettent de s'assurer de la stabilité des conditions opératoires.

\section{b) Instrumentation "diphasique" (Fig. 5)}

Elle comporte également environ 40 capteurs destinés principalement aux mesures suivantes:

- niveau liquide instantané ou moyen en régime stralifié (sondes capacitives)

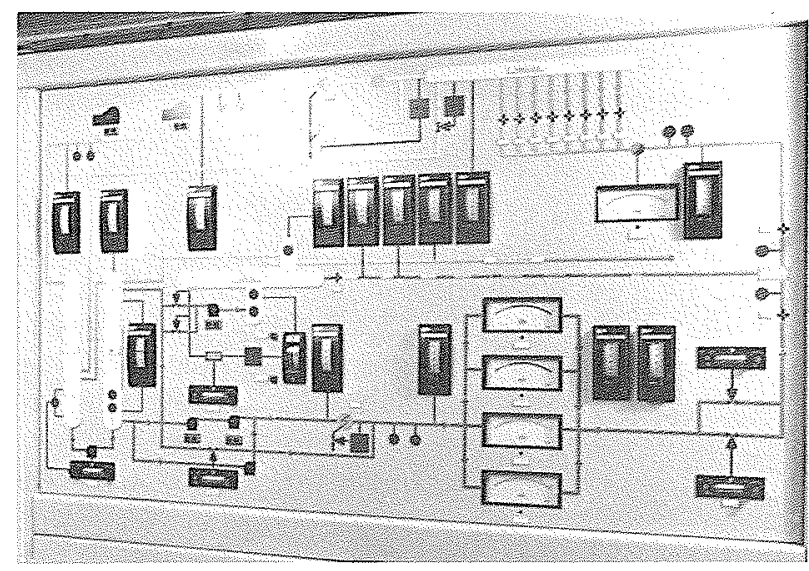

Figure 4 - Tableau synoptique en salle de contrôle.

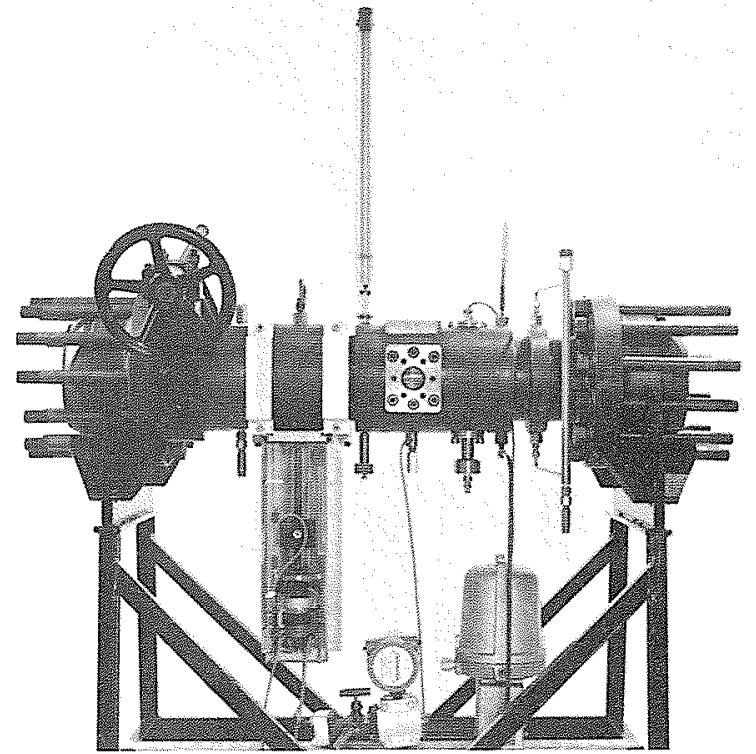

Figure 5 - Tronçon de mesures à brides toumantes.

- fraction liquide moyenne (gamma-métrie)

- détection de phase en paroi et taux de vide local (sondes à fibres optiques A.I.D.)

- pression différentielle moyenne (haute sensibilité).

Une partie des capteurs est repartie le long de la conduite d'essais afin de contrôler l'établissement du régime d'écoulement. Mais la majorité est regroupée en aval de la conduite d'essais sur un tronçon de mesure à brides tournantes équipé de hublots d'observation qui permet d'étudier l'écoulement sous différents axes. Ces capteurs, placés dans un environnement sévère et fonctionnant à leurs limites de sensibilité, ont nécessité de nombreuses mises au point. Ils sont contrôlés par des étalonnages fréquents.

\section{c) Acquisition et traitement des données}

L'ensemble de l'instrumentation est contrôlé par un système d'acquisition des données Hewlett-Packard 3052 A comportant notamment une unité centrale programmable en langage HPL de $24 \mathrm{k}$ mots, une unité d'échantillonnage à 80 voies de mesure (120 mesures/s séquentielles), un voltmètre numérique précis (7 chiffres) pour les capteurs lents et un voltmètre rapide (4500 mes./s à $31 / 2$ chiffres) pour l'étude des signaux rapides. Le système gère et programme les mesures, effectue les calculs d'interprétation en temps réel et fournit les "listings" des résultats. Il est complété par des enregistreurs graphiques et magnétiques qui permettent des traitements numériques en différé.

\section{Etude des écoulements diphasiques pétroliers}

Les premiers essais effectués ont porté sur les écoulements diphasiques à forte proportion de gaz, en vue de l'application au transport des gaz à condensats et bruts à forts "GOR". Les fluides, expérimentés sous 15 et 40 bars, sont un gaz naturel à $90 \%$ de méthane et une 


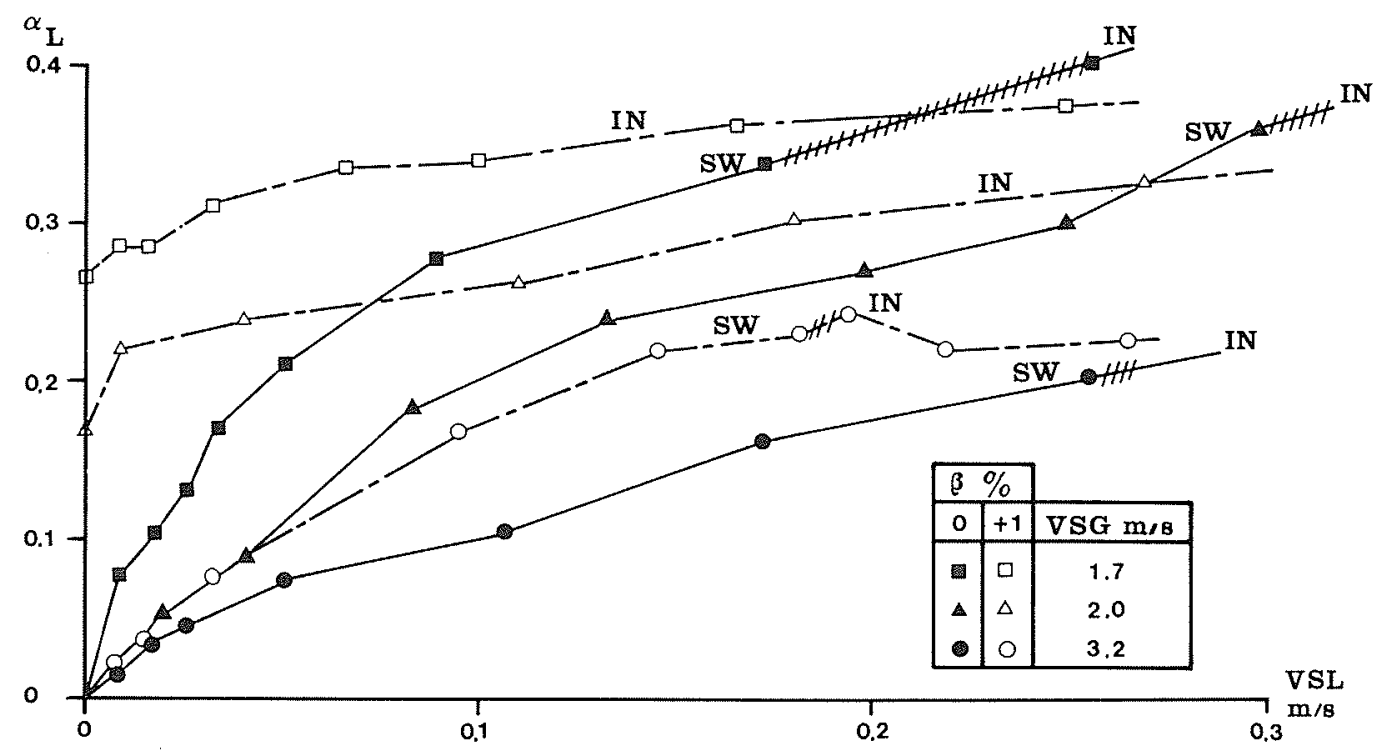

Figure 6 - Contenu liquide dans la conduite en horizontal et incliné à $1 \%$.

gazoline légère de densité 0,68 en conditions de stockage.

Les pentes étudiées sont comprises entre 0 et $1 \%$. Dans ces conditions, le régime d'écoulement stratifié prédomine, mais on peut également observer, pour des vitesses des fluides convenables, les régimes à poches et annulaire-dispersé.

En écoulement stratifié, les mesures essentielles sont celles de la fraction de liquide en place dans la conduite et de la perte de charge moyenne. Pour une pression et une pente données, elles peuvent être représentées en fonction des vitesses débitantes du gaz VSG et du liquide VSL.

\section{Exemples d'évolution pour des pentes de 0 et $+1 \% \dot{a}$ 15 bars [1]}

\section{a) Fraction liquide $\alpha_{L}$ (Fig. 6)}

En horizontal, $\alpha_{L}$ croît très rapidement de manière parabolique avec VSL de 0 jusqu'à une valeur toujours supérieure à la fraction liquide débitante $V S L /(V S L+$ $V S G)$, ce qui traduit une rétention du liquide. $\alpha_{L}$ diminue lorsque $V S G$ est augmenté à $V S L$ constant.

En incliné, le même comportement subsiste aux fortes valeurs de VSG. Par contre, au-dessous d'une valeur $V S G_{C E}$ située à $2,7 \mathrm{~m} / \mathrm{s}$ environ, dite "vitesse critiques d'entrainement", on constate une très forte accumulation de liquide aux faibles valeurs de VSL : lorsque $V S L \rightarrow 0$, il reste une fraction de liquide très importante dans la conduite, due à la création des conditions d'équilibre d'une nappe liquide en régime stratifié, ou à la formation de bouchons de liquide instables en régime à poches ; l'évolution de $\alpha_{L}$ à partir de sa valeur d'origine en fonction de $V S L$ est alors lente et d'allure rectiligne.

\section{b) Perte de charge DP/DX (Fig. 7).}

Les évolutions sont tributaires du contenu liquide $\alpha_{L}$.

En horizontal, les variations avec $V S L$ sont d'allure rectiligne. On vérifie par calcul que lorsque $V S L \rightarrow 0$, la perte de charge est conforme aux lois de l'écoulement monophasique du gaz.

En incliné, pour $V S G>V S G_{C E}$, le comportement est voisin de celui observé en horizontal, mais la croissance de $D P / D X$ avec $V S L$ est plus rapide. Pour $V S G<$ $V S G_{C E}, D P / D X$ présente une valeur initiale de l'ordre de 10 fois supérieure à celle de l'écoulement monophasique du gaz. Mais son augmentation en fonction de VSL est ensuite plus lente. Les représentations $D P / D X$ à différents $V S G$ se croisent. Il existe donc un minimum dans l'évolution de la perte de charge en fonction du débit global pour un système diphasique de composition VSG/ $V S L$ fixée.

\section{c) Régime d'écoulement (Fig. 6)}

La transition du régime stratifié au régime à poches ne fait pas apparaître de discontinuité notable dans les évolutions de $\alpha_{L}$ et $D P / D X$.

En horizontal, le régime stratifié ne dépend pas de manière nette de VSG (transition vers $V S L \simeq 0,25 \mathrm{~m} / \mathrm{s}$ ).

En incliné, pour $V S G>V S G_{C E}$, le domaine du régime stratifié semble légèrement diminué (transition vers $V S L \simeq 0,18 \mathrm{~m} / \mathrm{s}$ ). Pour $V S G<V S G_{C E}$, il disparait ici totalement.

\section{Confrontation avec les méthodes prévisionnelles classiques}

De nombreuses méthodes ont été proposées pour calculer la fraction liquide dans la conduite et la perte de charge. Elles ont été décrites en particulier dans l'analyse bibliographique des études $[2,3,4]$. On a testé principalement les méthodes de Beggs [5], Bonnecaze [6], Dukler-Eaton $[7,8]$, classiques dans l'industrie pétrolière, qui reposent sur des équations de type monophasique et des corrélations de fraction liquide et coefficients de frottement. On remarque (Fig. 8 et 9 ) la dispersion de ces différentes méthodes et leur manque de précision dans le cas d'une pente de $1 \%$ et d'une vitesse du 


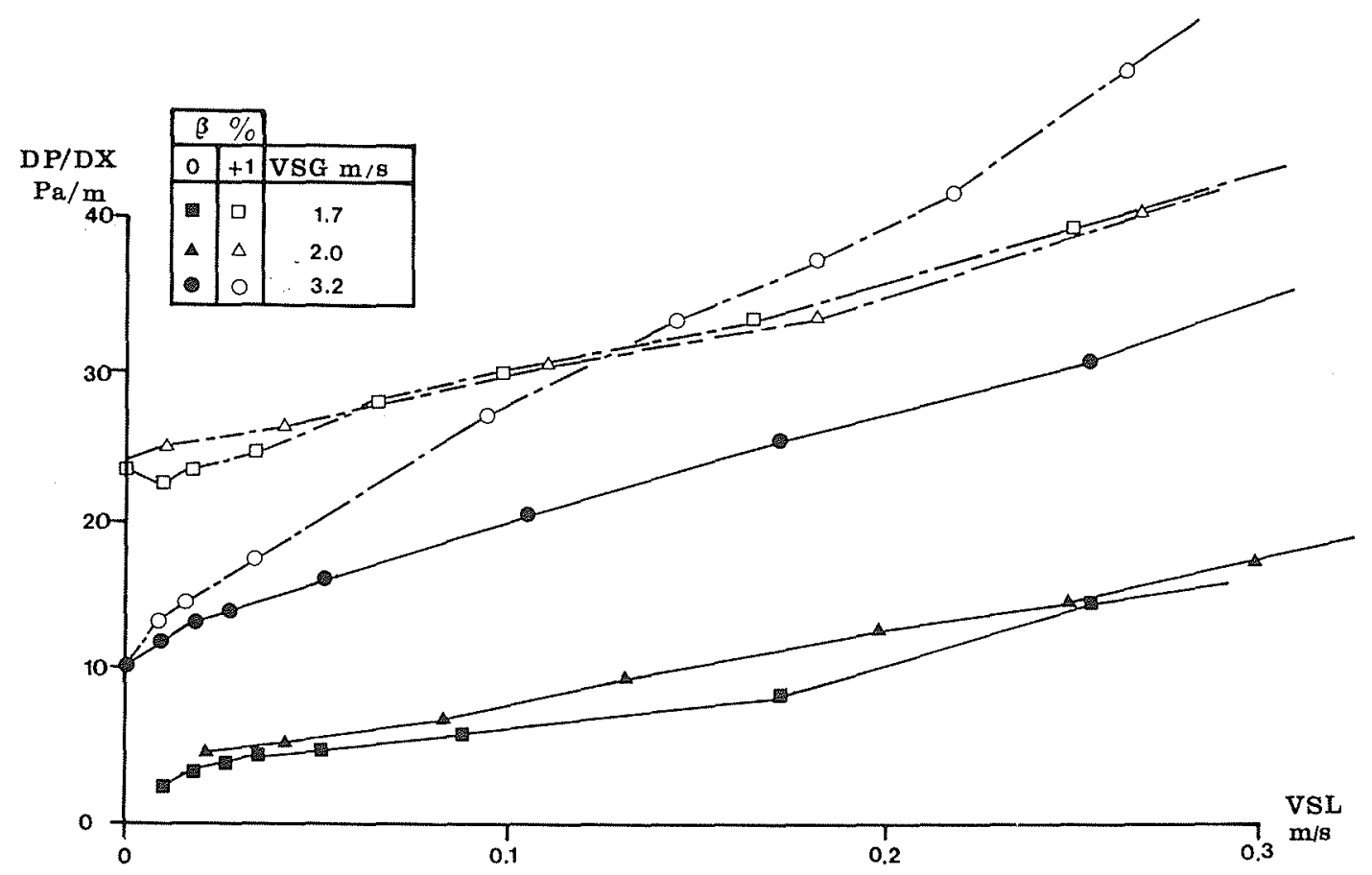

Figure 7 - Gradient de pression en horizontal et incliné à $1 \%$.

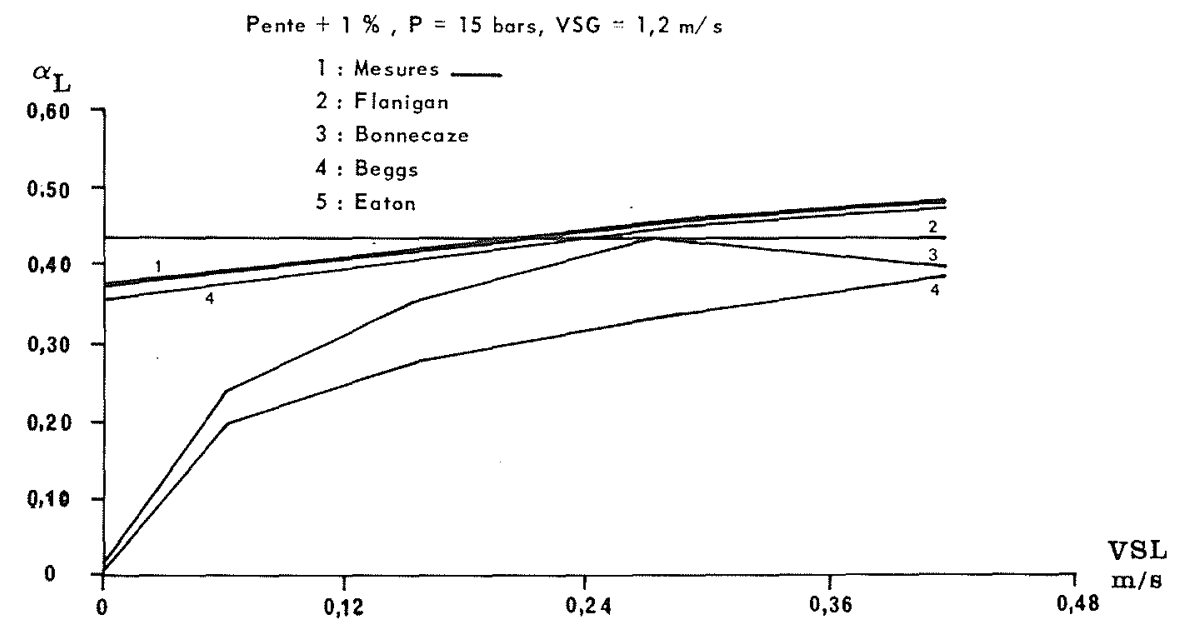

Figure 8 - Fraction liquide dans la conduite : Test des modèles classiques.

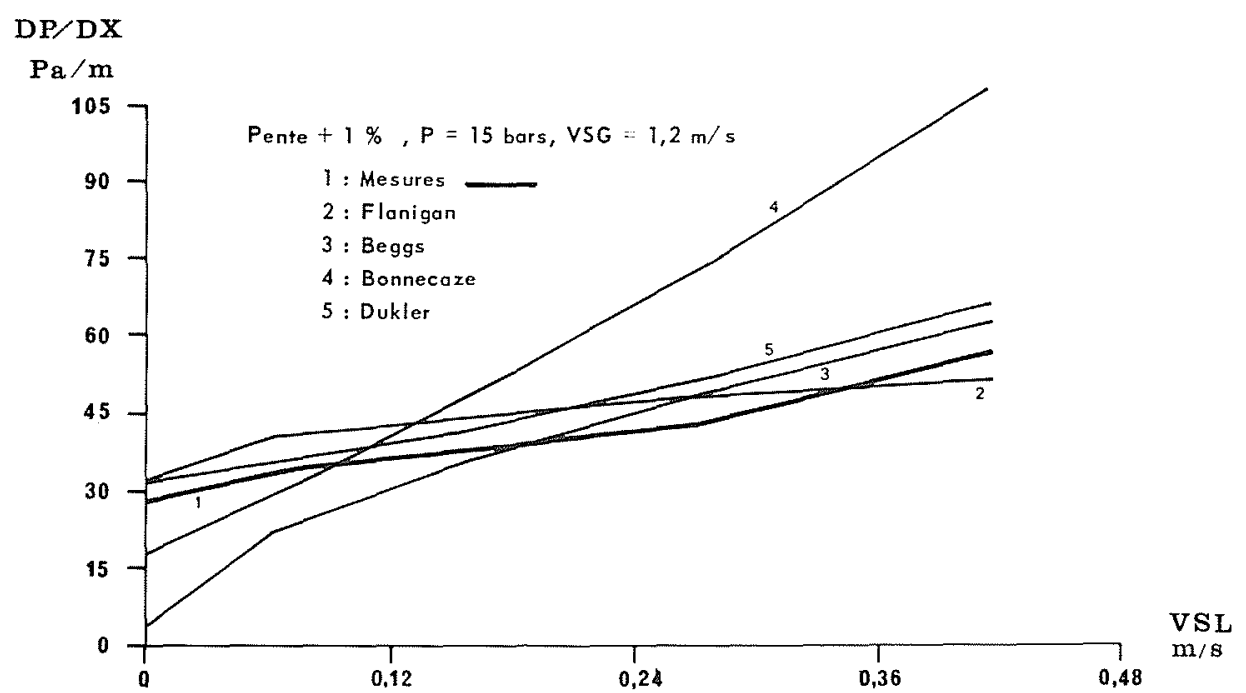

Figure 9 - Gradient de pression : Test des modèles classiques. 
gaz inférieure à $V S G_{C E}$. En configuration horizontale ou en ascendant lorsque $V S G>V S G_{C E}$ les résultats s'améliorent mais restent encore insuffisamment précis.

\section{Conclusion}

La technique expérimentale, brièvement exposée, permet de réaliser les nombreuses mesures nécessaires à l'exploration systématique du vaste domaine paramétrique défini en particulier par la pente, les vitesses superficielles des deux phases et leurs caractéristiques thermodynamiques. Outre les mesures globales présentées, on cherche à obtenir par des mesures plus fines, dont la précision doit être progressivement améliorée, des informations complémentaires nécessaires en particulier pour l'étude des écoulements à poches : notamment forme des interfaces, fluctuations de pression, présence éventuelle de bulles ou de brouillards.

L'interprétation des résultats ne peut se faire à partir des modèles trop simples dont l'exemple d'application a été présenté. Pour les écoulements stratifiés, on a retenu le modèle à écoulement séparé des deux phases et égalité des pertes de charge dans chaque phase. Les équations de bilan massique et quantité de mouvement sont classi- ques, mais la difficulté est de décrire convenablement les caractéristiques des périmètres "mouillés" et des interfaces. Cette manière de procéder, qui donne simultanément, en solution du système d'équations, la perte de charge et la fraction liquide en place, doit aussi permettre de déterminer la vitesse d'entrainement critique du gaz dont les implications sur les pertes de charge, la rétention du liquide et les instabilités sont très importantes.

\section{Références}

[1] MOULY P. - Thèse Docteur-Ingénieur, Paris VI, Avril 1979.

[2] FERRE D. - Thèse troisième cycle, PARIS VI, mai 1978

[3] CROUZIER O. - Thèse troisième cycle, PARIS VI, septembre 1978

[4] HADJ-ADMED A. - Thèse Docteur-Ingénieur, TOULOUSE, octobre 1978

[5] BEGGS H.D. BRILL J.P. - J.P.T, 25, 607 (1973)

[6] BONNECAZE R.H., ERSKINE W., GRESKOVICH E.J. AIChE J., 17, 5, 1109-1113 (september 1971)

(7) DUKLER A.E. et al. - Gaz-Liquid Flow in pipeline, Mono graph $N X-28$, HOUSTON University, May 1969.

[8] EATON B.A. et al. - J.P.T., 240, 815-829 (june 1967)

[9] FLANIGAN O. - Oil and Gas J., 56, 132 (march 10, 1958).

\section{Discussion}

Sur proposition du Président de Séance, M. J. DELACOUR, la discussion du mémoire de M. FERRE a été jointe à celle du mémoire de MM. CORTEVILLE, M. GROUVEL et Mme GROSSIN.

Le Président remercie M. FERRE et M. CORTEVILLE et ouvre la discussion.

M. DELHAYE - - Quelle est la signification de la courbe $\Delta p / \Delta x=f(R e)$. Comment explique-t-on l'allure de la courbe ? Qucllc est la définition du nombre de Mach? Quelles valeurs prend-il ?

M. FERRE. - Dans cette représentation, le coefficient de frottement $\Lambda$ prend en compte tout le gradient de pression expérimental. Il est corrélé à un nombre de Reynolds et à un nombre de Mach diphasique. Le modèle, qui suppose toute la perte de charge produite dans les bouchons, surestime donc ce coefficient de frottement dans les bouchons.

M. DELHAYE. - Que se passe-t-il quand on augmente le nombre de Reynolds?

M. FERRE. - D'après ROS, la courbe se poursuit selon une asymptote horizontale. La forte réduction de $\Lambda$ observée ici aux forts nombres de Reynolds provient, à mon avis, de la présence des bulles dans les bouchons qui réduit fortement le frottement à la paroi. Du moins est-ce une hypothèse.

M. DELHAYE. - Quelle est la précision obtenue sur la mesure du taux de vide en écoulement à poches? Quelle est la source utilisée ? Quelles sont la nature et les épaisseurs des parois à traverser ? Quels sont les temps et les taux de comptage ?

M. CORTEVILLE. - Nous effectuons actuellement les mesures en écoulements stratifiés avec une source de césium de 2 millicuries en intégrant le signal reçu pendant 30 secondes. La conduite est en acier d'épaisseur $30 \mathrm{~mm}$. La protection de plomb est de $7 \mathrm{~cm}$. Nous utiliserons prochainement une source de 30 millicuries pour permettre des mesures plus rapides.

M. DELHAYE. - Et vous arrivez à mesurer un taux de vide sur 2 millicuries avec le cesium?

$M$. ROUX. - Le tube comporte des fenêtres en polymère organique à faible absorption, dont l'épaisseur est juste suffisante pour tenir la pression.

Pour tenir compte de la variation logarithmique de l'absorption $\gamma$ lors des écoulements par bouchons, nous effectuons 30 comptages successifs d'une seconde. Un calculateur commande l'acquisition des données et effectue la moyenne logarithmique des 30 comptages.

Avec la source de $2 \mathrm{mCu}$, le comptage est d'environ 500 coups par seconde.

M. HULIN. - Quels résultats ont donné les sondes optiques dans des huiles un peu visqueuses?

M. CORTEVILLE. - Nous avons expérimenté des hydrocarbures très peu visqueux. Les sondes fonctionnent alors, bien qu'il subsiste des difficultés dues aux films liquides sur les parois.

M. JACQUEMIN. - Peut-on définir une longueur d'établissement lorsque la longueur des poches est voisine de celle de la conduite? Si on le pouvait quelle serait cette longueur et de quoi dépendrait-elle?

M. FERRE. - Dans le cas de poches très longues, l'écoulement est plus proche d'un écoulement stratifié que d'un écoulement à poches. Ne pouvant définir correctement la nature 
¿u régime, je ne peux non plus définir correctement cette longueur đ'établissement.

Dans les autres cas, on peut bien sûr définir cette longueur qui, dans mes conditions expérimentales, ne dépend pas des vitesses débitantes du gaz et du liquide.

M. JACQUEMIN. - Cette longueur d'entrée est-elle très différente de celle qu'on observe en écoulement verticàl ?

M. FERRE. - Pour les mêmes conditions d'entrée, l'écoulement à poche vertical semble s'établir plus rapidement.

M. BONIS. - Les débits critiques sont en dehors de votre domaine d'investigations, mais pouvez-vous dire ce que l'on observerait si l'on abaissait continuement la pression aval tout en maintenant la pression amont constante?

M. FERRE. - Je ne peux pas me risquer à vous répondre sur les conditions de débit critique en écoulement à poches.

M. BOUVARD. - J'aurais plusieurs questions à poser sur les résultats présentés.

Monsieur FERRE a présenté plusieurs résultats statistiques, dont l'un présente une forme polymodale (période des poches).

Est-ce que cette forme polymodale se maintient si vous augmentez le nombre d'essais ? Si non, elle résulterait d'un nombre d'essais trop réduit. Si oui, c'est qu'elle correspondrait à un fait physique que vous auriez pu en partie au moins cerner. Mais lequel ?

Dans une des formules présentées, on fait intervenir au dénominateur un terme $1-\mathrm{M}^{2}$ mais avec $\mathrm{M}$ de l'ordre de $15 \%$. Le terme résultant serait voisin de 1 (à $2 \%$ près environ). Est-il justifié de l'introduire compte tenu de la précision qu'on peut espérer obtenir dans l'interprétation des résultats certainement très difficile?

Vous avez fait état de réduction du frottement lorsqu'il y a des bulles d'air, et cette réduction est très importante. Est-ce que ce phénomène correspondrait à une réduction de la turbulence, par exemple du fait qu'il y a des poches qui gênent la transmission de fluctuation de pression?

Je vous signale d'autre part que nous avons constaté une réduction notable des fluctuations turbulentes sous l'influence de particules solides en suspension, dans des études menées à l'Institut de Mécanique de Grenoble.

Vous avez mesuré la coalescence des poches d'air. Est-ce que ce n'est pas le bilan que vous mesurez, entre la coalescence et la décoalescence? Ces deux phénomènes devraient intervenir simultanément, puisqu'il y a régime permanent si la conduite à une longueur très grande.

M. FERRE. - L'aspect polymodal de certains histogrammes n'est pas dû à un nombre trop faible de mesures. Il ne semble pas traduire un phénomène physique puisqu'il n'apparait aucun lien entre la présence de plusieurs modes et les régimes d'écoulement. Enfin, le test de conformité du $\chi^{2}$ valide (ou plutôt n'invalide pas) l'hypothèse d'histogrammes gaussiens ; 1'hypothèse d'un aspect polymodal réel m'apparaît donc comme étant statistiquement non fondé.

N'ayant fait aucune mesure, je ne peux savoir à priori quelles sont les actions réciproques de la turbulence et des bulles. Il serait certainement instructif d'entreprendre une telle étude dans le cas des écoulements confinés.

M. BOUVARD. - A Grenoble, dans des essais que nous avons fait, nous avons constaté qu'avec des matériaux solides, la turbulence se trouvait réduite. Nous l'avons constaté pratiquement, explicitement.

Est-ce un phénomène analogue que vous auriez avec des bulles d'air, ou est-ce quelque chose de différent qui réduirait la perte de charge.

M. FERRE. - Je ne peux ni infirmer, ni confirmer ces hypothèses, faute de mesures de vitesse locale.

M. le Président. - Question sur la coalescence et la décoalescence?

M. FERRE. - La coalescence apparaît comme étant l'évolution normale de l'écoulement lorsqu'il y a détente du gaz dans la conduite. Il y a réorganisation de l'écoulement pour suivre l'évolution de la vitesse locale du gaz.

Par ailleurs, je n'ai jamais observé de décoalescence dans l'installation.

M. DELHAYE. - Dans la représentation $\alpha_{L}=f\left(V_{S L}\right)$ comment a été mesuré $\alpha_{L}$ ? S'agit-il d'un taux de présence du liquide sur un diamètre ou sur une section droite?

A-t-on cherché à représenter les résultats dans un điagramme de Zuber et Findlay ?

M. CORTEVILLE. $-\alpha$ est un taux de vide moyenné sur une section droite. Il est déduit de mesures diamétrales, avec l'hypothèse que l'interface liquide-gaz est plan en régime d'écoulement stratifié. Nous comparons les valeurs de $\alpha_{L}$ et les domaines de régimes d'écoulements avec ceux prévus notamment par la méthode de TAITEL et DUKLER.

M. MEGNIN. - Qu'est-ce qui a présidé au choix du diamètre et quelle est l'incertitude sur l'horizontalité de votre conduite?

M. FERRE. - Le diamètre doit être suffisamment grand pour obtenir des écoulements semblables à ceux présents dans les installations industrielles ; $4 \mathrm{~cm}$ est un minimum. D'autre part, l'étude de la longueur d'établissement impose une conduite assez longue. La place disponible nous a alors limité à un diamètre de $4,5 \mathrm{~cm}$ et une longueur de $50 \mathrm{~m}$.

L'horizontalité est assurée à quelques secondes d'arc près.

M. SEMERIA. - Au sujet des distributions polymodales, j'ai obtenu dans les temps très anciens des courbes à deux bosses pour les écoulements à bulles.

Il $\mathrm{y}$ avait une population de bulles indépendantes d'une part, et d'autre part, une population de bulles résultant de coalescences venant de la première population. Une telle explication s'appliquerait-elle dans votre cas?

M. FERRE. - Pour moi, toutc poche résulte de coalescence.

M. SEMERIA. - Certaines poches se forment dès l'entrée, d'autres très loin de l'entrée. Cela peut faire deux familles.

M. FERRE - Effectivement, c'est une explication qu'il faudra approfondir. 


\section{Abstract}

\section{Experimental study of two-phase petroleum flow over rough ground}

Predicting two-phase behaviour in subhorizontal pipes has become critical for offshore oil and gas fields development, specially in great water depths or when the sea bed is uneven. Standard methods of calculating average pressure losses are lacking in precision and do not predict transient phenomena, e.g. liquid slugs length or separation of phases at low points in the lines. As a result, two-phase flow is generally ruled out as a method of transporting produced hydrocarbons to land, even though this process is very simple and requires only low investments.

The objective of the "two-phase flow research group", associating CFP, IFP and SNEA (P) within the GERTH, is to improve methods of predicting flow regimes, spatial phases distributions and pressure losses, specially in inclined pipelines.

After preliminary air-water experiments on a two inch test loop at the LMEF of PARIS-ORSAY, construction of a test loop working in industrial conditions was undertaken in 1977 at the SNEA (P) gas plant of BOUSSENS.

Main characteristics of this 6" loop are :

- Length of the testline : $400 \mathrm{ft}$ (260 ft in inclined position)

- Continuous variable slopes between $-10 \%$ and $+10 \%$ Average test pressure between 100 and 800 psi
- Superficial gas flow velocity between 0 and $30 \mathrm{ft} / \mathrm{s}$

- Superficial liquid flow velocity between 0 and $10 \mathrm{ft} / \mathrm{s}$. The loop is equiped with about 40 process instruments and 40 two-phase flow sensors such as slug detection probes or average liquid fraction probes. Instruments are monitored by a $3052 \mathrm{~A}$ H.P. data acquisition system which controls measurements in real time and print out lists of processed resuits.

First experiments were made at high GOR flows, in horizontal or slowly rising slopes, where the stratified flow pattern predominates. Results confirmed the strong influence of the inclination angle on pattern, liquid hold-up and pressure gradient, in particular at moderated gas flow rates. A critical gas velocity was observed at very high GOR in inclined flow: if the gas velocity is below this critical value, the liquid accumulates in the line, increasing drastically the pressure losses.

The experimental data were compared to the results of the predictive models usually used in oil industry. All these methods do not take enough account of the slope influence, especially for high GOR at gas flow rates below the critical gas velocity. So, theoretical research is going on, in order to develop a more precise stratified flow model. 